\title{
Suggestions on Discrimination and Training for Occupational Responsibility of Preschool Teachers
}

\author{
Xue $\mathrm{Li}^{\mathrm{a}}$, Liqiong Jiang \\ School of Pre-school Education of Chongqing University of Education, Chongqing, China, 400065 \\ alixue@cque.edu.cn
}

\begin{abstract} their occupational responsibility.

\section{FOREWORD}

People are committed to finding and summarizing the influencing factors and training measures of teacher performance since always. Due to the diffuse and persistent effects of responsibility in the educational work of teachers, the researchers have started to focus on the manifestation, characteristics and cultivation measures since the 1980s. With frequent child abuse by preschool teachers, sorting out the recent research progress of teachers' occupational responsibility with the clear discrimination of the occupational responsibility concept contribute to improving the preschool teachers' professional performance and occupational level.
\end{abstract}

The occupational responsibility of preschool teachers is an important measurement for their occupational level, and an indivisible unity composed of responsibility cognition, responsibility emotion and responsibility behaviors. Strengthening the rational research on preschool teachers' occupational responsibility, clarifying the range of their occupational responsibility cognition, arousing the correct and positive emotion of occupational responsibility, and paying attention to their occupational responsibility behaviors, etc. are conducive to training

Keywords: Preschool teachers; Occupational responsibility; Concept Cultivation

\section{COURSE AND CHARACTERISTICS OF RESPONSIBILITY RESEARCH}

After retrospecting the research on responsibility for many years, we have found out that it can be divided into three stages roughly:

First stage: sprouting period. The research on responsibility is contained in the moral education in this period. For example, the philosophers, Plato, Aristotle and Zeno, have begun to apply the implication of responsibility in their analysis of justice, duty, and punishment. In the opinion of Mekeon (1957), the responsibility formally appeared in English, German and French in the 17th or 18th century, and referred to (1) loyalty to the Pope or the parliament; (2) duty of performance; and (3) moral obligation [1]. In the perspective of Davis (1973), the responsibility in philosophical significance carried on as before mainly referred to negligence responsibility and moral responsibility [2]. Research characteristics of this period: (1) The research is very indistinct with rough outline. (2) The influence of responsibility on work performance has been focused on. (3) Moral discussion is obvious. (4) Although the basic theoretical problems of responsibility like connotation, objectives and cultivation paths have been discussed, they are hinted and require deep research still.

Second stage: exploring period. Since the 1980s, with the development and improvement of the Big Five Model of Personality, the responsibility has abstracted the researchers as one of dimensions and finally separated the responsibility from the moral category, becoming an independent research object. In this period, multiple theoretical problems involved in responsibility have been researched to a relatively comprehensive extent. After analyzing the wrongful conducts and acts of lying of children in daily life, Piaget indicated that people's responsibility is from the judgment for responsible behaviors in the childhood. The responsibility of preschool children is growing from the objective behavior responsibility idea based on consequences to the subjective responsibility idea based on behavioral motives and intentions. He called two responsible attitudes as "objective responsibility" and "subjective responsibility", and also indicated that the responsibility of children was gradually formed through internalization of external standards. Its general rule lies in the first appearance of objective responsibility followed by the growth of subjective responsibility. The former gradually steps into the subordinate position, but the latter gradually substitutes the former in the dominant position [3] Main features include: (1) Research has become more scientific and normative. Opinions on connotations of responsibility still remain controversial, but the responsibility is agreed to influence the work performance. All researchers have agreed that the responsibility is an integral whole with the influence of such characteristics as knowledge, emotion, and behaviors. (2) The connection between research and psychology is getting closer. A batch of scholars represented by Wang Jianmin [4] have started to explore the mental structure of responsibility to try to build the responsibility cultivation model with reasonable frame and obvious effect. (3) Research has begun to expand. With more and more attention paid by the researchers to responsibility, there are abundant related researches. For 
example, the academic responsibility has the predictive effect for study [5], etc.

The third stage: development period. Since the 1990s, the responsibility has frequently occurred in the periodicals and magazines as an independent research object. Main characteristics of research in this period include: (1) The research has been gradually theorized [6] [7]. Basic theoretical problems in the field of responsibility, such as meaning and characteristics of responsibility, have been studied scientifically. For example, the mental structure of responsibility raised by the researcher includes three aspects, including responsibility cognition, responsibility emotion and responsible behaviors. (2) The research has been gradually diverged. The branch development of responsibility has been studied, such as occupational responsibility, academic responsibility and social responsibility [8]. The mesomeric effect on responsibility has been concerned. Kochansha and colleagues have found out in the research that the responsibility is from the early obedience of children to parents' requirements. In their opinion, when the children follow and actually practice those arranged by their mother, it seems to an early sign of responsibility internalization [9]. Harris has known the responsible behaviors of children by peer evaluation method, and found out that the responsible behaviors have higher positive correlation to the responsibility judgment level [10]. (3) Empirical research has been introduced [11]

\section{DISCRIMINATION OF OCCUPATIONAL RESPONSIBILITY}

\subsection{Understanding about connotations of occupational responsibility}

After analyzing multiple responsibility concept definitions, we have found the following problems at present: (1) The understanding about the connotations of occupational responsibility is too narrow. This is mainly reflected in that some researchers replace the responsibility with a sense of responsibility and the responsibility consciousness. To some extent, the occupational responsibility is more of a subjective cognition and experience, so it has a certain degree of rationality. However, the occupational responsibility behaviors mark the level of occupational responsibility. Ignoring the explicit function of occupational responsibility behaviors shows the limitation of its definition. (2) The positioning of occupational responsibility is not clear. For example, some researchers have oriented the occupational responsibility as a psychological state. As everyone knows, the mental state is relatively stable and continuous that the individual's mental activity appears in a period [12]. Although the occupational responsibility will lead to a relatively stable psychological state in the process of performing social responsibility behaviors, this state is the result and the occupational responsibility is the reason. Taking the effect for the cause is obviously wrong.
In our opinions, (1) the connotations of occupational responsibility shall be the measurement for the level of psychological activities of responsibility, and the denotation shows the level of various psychological activities of responsibility in work. (2) The occupational responsibility is not only a one-dimensional explicit behavior characteristic or responsibility consciousness. It shall be the integration and unification of potential components such as occupational responsibility behavior tendency, sense of occupational responsibility, occupational responsibility concept, and explicit occupational responsibility behaviors. (3) When individuals form the occupational responsibility concept and emotion and execute its behaviors, it is under the reference to the code of professional responsibility. Moreover, the code of professional responsibility is formed during the social development. The social culture has an important influence and effect on the formation of occupational responsibility.

\subsection{Division of occupational responsibility}

In our opinions, the occupational responsibility will be manifested in three aspects, namely individual's cognition, emotion and behaviors from the classification of psychological dimension characteristics or the responsibility relation object division.

The occupational responsibility cognition is manifested in the individuals' behaviors and activities, and is the cognitive characteristic factor directly influencing the mechanism and level of responsibility behaviors. It is the basis of individual occupational responsibility level and determines the level of occupational responsibility to some extent. It decides the occupational responsibility of teachers by solving "believe it or not" in the teachers' thought. To some extent, the occupational responsibility cognition determines the level of occupational responsibility. Occupational responsibility cognition can be divided into organization and process. The organization refers to the individual's occupational responsibility knowledge. It restricts the scope and principle of individuals for occupational responsibility cognition, and constitutes the carrier of process. The process refers to all cognitive processing of individuals through social interaction for these occupational responsibility knowledge. It is the final cognitive outcome of individuals, and the premise and power for emotions and behaviors. Specifically, it can be manifested as: preschool teachers' cognition and understanding; information processing and analysis capabilities of preschool teachers for responsibility situations; and preschool teachers' prediction of their own abilities and the significance of responsibility event consequences.

The occupational responsibility emotion, also called the sense of occupational responsibility, refers to the emotional experience of individuals during responsibility behaviors. It is mainly accompanied by the conflict (consistency or inconsistency) between the individual's cognitive factors and behavioral factors. It restricts the course of individual cognitive processing, and determines the occurrence and development of responsibility behaviors. It influences the 
overall benign enhancement of occupational responsibility by solving "willing or not" in the emotion of preschool teachers. Specifically, it can be manifested as: preschool teachers' professional self-identity; degree of acuity of the preschool teachers for emotional experience in responsibility events; and role of preschool teachers' emotions in promoting responsibility behaviors.

Occupational responsibility behaviors refer to a series of behavioral patterns taken by individuals to achieve a certain purpose of occupational responsibility. It is the explicit result under the combined action of individual occupational responsibility cognition and emotion, and embodies the level of occupational responsibility in a centralized way. Specifically, it can be manifested as: whether the preschool teachers take the initiative to undertake or accept the responsibility tasks voluntarily; whether the preschool teachers can overcome internal and external difficulties and persist to the realization of objectives with sufficient energy and perseverance while fulfilling the responsibility; how can preschool teachers monitor and regulate their own professional behaviors in order to achieve a certain responsibility; and whether the preschool teachers make decisions independently, execute decisions and have their own ideas without dependence on others in the process of responsibility behaviors.

\section{ENLIGHTENMENT FOR EDUCATIONAL PRACTICE}

\subsection{Improvement of research means, and strengthening of rational research on occupational responsibility}

The responsibility is an integrative concept involving psychological states, mental activities, etc., and is influenced by social culture, individual experiences, educational level and other factors. It is impossible to better understand the complete picture of occupational responsibility by such means as current factory analysis. It is necessary to improve or introduce new research means, develop the qualitative and quantitative research methods, and deepen the research on the occupational responsibility itself, so as to assist the researchers in making scientific advice or guidance. Besides, the occupational responsibility of preschool teachers have common requirements of the industry, and also unique occupational requirements of preschool teachers. The integration of protection and teaching is an important feature of educational work of preschool teachers. In kindergarten, the preschool teachers should take comprehensive responsibility for the whole activity of preschool children. They should not only take care of the daily life, diets and sleep of preschool children, guide their physical exercise and care for their physical and psychological health, but also they should direct diversified activities like games and study to promote their growth in intelligence, emotion, sociality, etc. The work of preschool teachers should be very meticulous. Preschool children have poor ability to live independently. Teachers should elaborately take care of their lives, such as feeding preschool children and assisting them in rolling up sleeves. Subtle changes in physical health or emotion of preschool children should be found out upon the careful observation of preschool teachers and timely solved. So, the preschool teachers play the roles of mother, teacher and friend.

\subsection{Clarification for scope of preschool teachers' occupational responsibility cognition}

The occupational responsibility cognition is the basis of individual occupational responsibility level and determines the level of occupational responsibility to some extent. The occupational responsibility cognition of preschool teachers can be enhanced from the clear discrimination of preschool teachers' responsibility scope and the improvement of occupational responsibility evaluation ability. The occupational responsibility cognition of preschool teachers can be enhanced from the clear discrimination of preschool teachers' responsibility scope and the improvement of occupational responsibility evaluation ability.The "scope of occupational responsibility is too generalized and narrowed" at present, resulting in the disorder of processing in reality. One locates the occupational responsibility of preschool teachers in an idealized and messianic holy position. Blind raise of occupational responsibility will easily lower the self-efficacy of the preschool teachers, resulting in the act of evading a teacher's responsibilities. The other fuzzes up and abstracts the occupational responsibility of preschool teachers. Too roughly narrowed occupational responsibility of preschool teachers cannot be expressed to scientifically and effectively guide the purpose of educational practice. The teacher training institutions should pay attention to the accumulation of teachers' real experience on the one hand, and promote the establishment of their rational reflection on the other hand. The effective rational reflection will further promote the formation of preschool teachers' responsibility idea and finally sublimate the moral personality of teachers. The effective rational reflection not only includes the summarization, conclusion and promotion of experience, but also includes the evaluation on activity process and activity results of preschool children. The trainer can direct the reflection by asking the appropriate questions: for example, what have you just experienced? How is it happen? What happens in another way? etc. The trainer may also state what have learned from the experience by guiding the preschool teachers to write the analytical paper, and improve the reflective evaluation ability of preschool teachers for occupational responsibility by means of evaluation on selfperformance, etc.

\subsection{Evocation of preschool teachers' positive and correct occupational responsibility emotion}

Correct and positive emotional experience of occupational responsibility will influence the processing course of teachers' occupational responsibility cognition, and it will 
become an internal impetus to promote the teachers to have occupational responsibility behaviors or to stop the irresponsible behaviors with the occupational responsibility cognition. The evocation of occupational responsibility emotion is a cultural identity substantially. The preschool teachers should explore the meaning and direction of teachers' occupational responsibility in the responsibility scenarios for emotional qualitative change of occupational responsibility. With deeper touch of occupational responsibility, the occupational responsibility behaviors will be executed more conscientiously. The evocation of occupational responsibility emotion is a scenarized cultural identity substantially. The teacher training institutions should strive to build the scenarized culture atmosphere of respect and trust. The management organization shall build the cultural atmosphere of respect and trust, lead the preschool teachers to have the emotional resonance by setting a clear image of models and vivid examples, and strengthen the empathy training, etc. conducive to arousing the occupational responsibility emotion. The following items shall be focused on regarding the establishment of preschool teachers' responsibility scenarios: (1) Accord with the age and occupation characteristics of preschool teachers. The scenarios should be set from the perspective of preschool teachers, and started from their study demands and contents. (2) Cause the cognitive conflict of preschool teachers. This is because that a moral behavior of an individual is absolutely not a simple problem, but it is a complex and nonlinear process. Therefore, the scenarios set shall include contradictions and conflicts. Analyzing, sorting out and solving the contradictions and conflicts will assist the preschool teachers out of confusion and bewilderment.

\subsection{Attention paid to practical guidance of preschool teachers' occupational responsibility behaviors}

The occupational responsibility behaviors are explicit results of individual responsibility cognition and combined action of responsibility and emotion, and have embodied the level of occupational responsibility in a centralized way. The final objective for training the preschool teachers about occupational responsibility is also the change of their occupational responsibility behaviors. The research shows that the practical activities play a very important role in the course of training the teachers' occupational responsibility behaviors. Therefore, With attention paid to the guidance and evaluation of preschool teachers in practice work situations, it is required to pay attention to the guidance on the practical work situations of preschool teachers, and strengthen the cultivation for self-control capability to finally lead to occupational responsibility behaviors and form good occupational responsibility. The scientific practice evaluation will promote the preschool teachers to examine their own responsibility behaviors reasonably, so as to improve their selfefficacy and solve the problem of "do or not do" in behavior. The occupational responsibility of preschool teachers reflects the psychological and behavioral characteristics and tendencies of preschool teachers for the degree of responsibility undertaken by the education employees, and it is a complete mental structure including knowledge, emotion, behaviors and other factors. The preschool teachers with different levels of occupational responsibility will be diversified in three aspects, including responsibility cognition, responsibility emotion and responsibility behaviors. The education researcher should further consider the ways to promote the preschool teachers' occupational responsibility by an integrative teaching method and finally boost their work performance.

\section{CONCLUSION}

5.1 The research course of responsibility includes sprouting period 、exploring period and development period.

5.2 The occupational responsibility shall be the measurement for the level of psychological activities of responsibility, and shows the level of various psychological activities of responsibility in work.

5.3 The occupational responsibility is a comprehensive unity of occupational responsibility cognition 、 occupational responsibility emotion and occupational responsibility behavior.

5.4 The rational research of occupational responsibility should be strengthened. Preschool teachers' occupational responsibility should be enhanced from the following aspects: clarifying the cognitive category of occupational responsibility, arousing the correct emotion of occupational responsibility and attaching importance to the practical guidance of occupational responsibility behavior.

\section{ACKNOWLEDGEMENT}

This paper is one of the achievements of key unfunded topic in the "13th Five-year Plan" of educational science in Chongqing, Research on Development and Application of Advanced Course Resources under Core Visual Threshold (2019-GX-133). One of construction achievements of the university-level research platform of Chongqing University of Education, "Family Education Research Center for Children Aged 0-6"(16xjpt03).

\section{REFERENCES}

[1] Jennifer S. Lerner and Philip E. Tetlock. Accounting for the effects of accountabili-ty. Psychological Bulletin, 1999, 125(2): 255-275 
[2] Barry R. Schlenker, Thomas W Britt. The Triangle model of responsibility. Psychological Review, 1994, 101(4): 632-652

[3] Li Boshu and Cen Guozhen. Moral Development and Mode of Moral Education. Shanghai: East China Normal University Press, 1999

[4] Wang Jianmin. Experimental Research Report on Formation of Children's Social Three-dimensional Structure (1996). Psychological Development and Education, 3:12-18

[5] Yan Guocai. Further Discussion on Learning Theories for Combination of Intelligence and Nonintelligence Factors (1995). Jiangxi Educational Research, 3:31-34

[6] Li Ming and Ye Haosheng. The Multiple Connotation and Structure of Responsibility and the Relevant Theoretical Integration (2009). Psychological Development and Education, 3:123-128
[7] Zhang Jijia. Discussion on Mental Structure of Responsibility (1998). Educational Research and Experiment, 4:43-47

[8] Li Xue. Research on Social Responsibility Structure and Its Development Characteristics of Middle School Students (2004), Master's thesis of Southwest China Normal University:5-15

[9] Kochanska, G. \&Aksan, N. Mother-child mutually positive affect, the quality of child compliance to requests and prohibitions and maternal control as correlations of early internalization. Child Development, 1995, 66: 236-254

[10] Harris, B. Developmental differences in the attribution of responsibility. Developmental Psychology, 1977, 13: 257-265

[11] Sheldon K M, Schachtman T R. Obligations, Internalization, and Excuse Making Integrating the Triangle Model and Self-Determination Theory. Journal of Personality, 2007, 75(2):359-382

[12] Huang Xiting. Introduction to Psychology (1991). Beijing: People's Education Press, Chapter I: Introduction: 1-10 